\title{
Left Ventricular Velocity of Propagation: A Useful Non- Invasive Measurement When Assessing Hemodynamic Alterations in Pulmonary Arterial Hypertension
}

\author{
Dagmar F. Hernandez-Suarez ${ }^{\mathrm{a}}$, Denada Palm ${ }^{\mathrm{b}}$, Francisco Lopez-Menendez ${ }^{\mathrm{b}}$, \\ Marcel Mesa Pabon ${ }^{\mathrm{c}}$, Angel Lopez-Candales ${ }^{\mathrm{c}, \mathrm{d}}$
}

\begin{abstract}
Background: Left ventricular (LV) velocity of propagation (Vp) has been shown to be inversely related to the LV relaxation time constant. We sought to examine $\mathrm{Vp}$ from a group of chronic pulmonary hypertension (cPH) patients and compare these values to $\mathrm{Vp}$ obtained in normal individuals and patients with known LV diastolic dysfunction (LVDD).

Methods: Echo-Doppler data and Vp measurements were retrospectively collected from all patients. The studied population was divided into four groups. Group I comprised of 15 patients with normal LV diastole, group II included 27 patients with stage 1 LVDD, group III was made up of 27 patients with stage 2 LVDD, and group IV included 66 patients with $\mathrm{cPH}$.

Results: In the $\mathrm{cPH}$ population studied, patients had smaller enddiastolic LV cavities with the highest $\mathrm{Vp}$ values but their early mitral inflow to $\mathrm{Vp}$ ratios were not different from healthy controls. In addition, $\mathrm{Vp}$ values and pulmonary wedge capillary pressures were significantly associated in patients with LV dysfunction or pulmonary hypertension $(\mathrm{P}<0.01)$.

Conclusions: LVVp might be a useful non-invasive measurement to be routinely obtained in $\mathrm{cPH}$ patients as it probably not only reflects the compressive forces being exerted on the LV, known to increase $\mathrm{Vp}$, but also might be quite useful for the non-invasive assessment of pulmonary capillary wedge pressures in these patients.
\end{abstract}

Keywords: Cardiac hemodynamics; Echo-Doppler; Left ventricle;

Manuscript accepted for publication April 03, 2017

${ }^{a}$ Medicine Division, University of Puerto Rico School of Medicine, San Juan, Puerto Rico

${ }^{b}$ Division of Cardiovascular Health and Diseases, University of Cincinnati College of Medicine, Cincinnati, OH, USA

${ }^{\mathrm{c}}$ Cardiovascular Medicine Division, University of Puerto Rico School of Medicine, San Juan, Puerto Rico

${ }^{\mathrm{d} C}$ Corresponding Author: Angel Lopez-Candales, Cardiovascular Medicine Division, University of Puerto Rico School of Medicine, Medical Sciences Building, PO Box 365067, San Juan 00936-5067, Puerto Rico.

Email: angel.lopez17@upr.edu

doi: https://doi.org/10.14740/cr541w
Right ventricular dysfunction; Relaxation; Propagation velocity

\section{Introduction}

The physiology of normal left ventricular (LV) early-diastolic filling not only is regulated by indices of active relaxation and passive chamber stiffness, but also by principles of fluid dynamics [1-3]. Even though elastic recoil and myocardial relaxation rate determine LV early-diastolic performance, the integrity of LV synchrony and geometry is essential to maintain the effect of their timely action on early-diastolic LV filling [4]. These factors are not only prime determinants of LV pressure decay during isovolumic relaxation and immediately after mitral valve opening, but they also instigate the generation of a sufficient intra-ventricular pressure gradient, which enhances efficient early-diastolic LV filling [4]. Accurate non-invasive echo-Doppler assessments of LV diastolic abnormalities have important therapeutic and prognostic implications. However, at times it remains a potential challenge for many cardiologists, particularly when evaluation of LV relaxation using standard Doppler echocardiographic parameters is hindered by their preload dependency [5].

Color M-mode of the mitral inflow displays color-coded mean velocities of the blood column as it propagates from the left atria to LV. This velocity of propagation (Vp) not only is inversely related to the LV relaxation time constant, but also is a largely preload independent index of LV diastolic performance [6-12]. Color M-mode Vp data have been studied both in animals and humans [13] and found particularly useful to identify patients with varying degrees of LV diastolic dysfunction (LVDD) [12-16].

Both magnitude and vector of the pressure gradient formation are dependent on LV geometry. Specifically, apical to base negative gradient formation is enhanced in small ellipsoidal LV cavities and corresponding $\mathrm{Vp}$ values higher than in normal sized ventricles, as blood redistribution by vortex formation and propagation requires that the LV diameter sufficiently exceeds the mitral diameter [17-20].

Since the pressure-overloaded right ventricle (RV), as a result of pulmonary arterial hypertension (PAH), not only is known to significantly alter LV geometry [21], but also impair 
LV filling based on cardiac magnetic resonance data [22], we sought to study if there were any differences in $\mathrm{Vp}$ values obtained from PAH patients when compared to patients with normal LV diastolic function as well as with patients with stage 1 and 2 LVDD.

\section{Methods}

\section{Population studied}

Study approval was obtained from the University of Cincinnati IRB Office (protocol number 12061302) and since this was a retrospective study, there was no need to obtain a written consent.

For the purpose of this study, our echocardiographic database at the University of Cincinnati was queried for transthoracic echocardiograms in which color M-mode acquisition was performed across the mitral valve from the four-chamber apical view. Consecutive studies were reviewed and for final inclusion into the study, all patients had to be in normal sinus rhythm at the time of the examination. In addition, a complete spectral Doppler study was needed to examine LV diastolic function in accordance with American Society of Echocardiography guidelines $[16,23]$. In contrast, data from patients with atrial fibrillation, frequent premature or atrial contraction beats were excluded. Patients with LVDD stage 3 were also excluded, as most of subjects falling into this category have significant LV systolic dysfunction which could be an important cofounder in our study.

\section{Echocardiographic studies}

Two-dimensional echocardiographic studies were performed using commercially available systems (Vivid 7 and 9; GE Medical Systems, Milwaukee, WI, USA). Images were obtained in the parasternal and apical views with the patient in the left lateral decubitus position and in the subcostal view with the patient in the supine position using a $3.5 \mathrm{MHz}$ transducer. Standard two-dimensional, color, pulsed, and continuous-wave Doppler data were digitally acquired in gently held end-expiration, and saved in regular cine loop format for subsequent offline analysis.

The following parameters were measured. 1) LV endsystolic and end-diastolic volumes were traced from the apical four-chamber view in accordance to published data, while ejection fraction calculations were done using the Simpson's rule algorithm [23]. 2) Mitral inflow velocities were obtained using pulsed-wave Doppler examination at a sweep speed of $100 \mathrm{~mm} / \mathrm{s}$ from the apical four-chamber view by placing the sample volume at the tips of the mitral leaflets and peak early-diastolic (E-wave) velocity was measured as previously described [16]. 3) In terms of tissue Doppler imaging (TDI) of the lateral portion of the mitral annulus (MA), early-diastole $\left(E^{\prime}\right)$ and late-diastole $\left(\mathrm{A}^{\prime}\right)$ velocities were measured by placing the sample volume at the junction where the mitral valve plane intersects the LV free wall using images obtained from

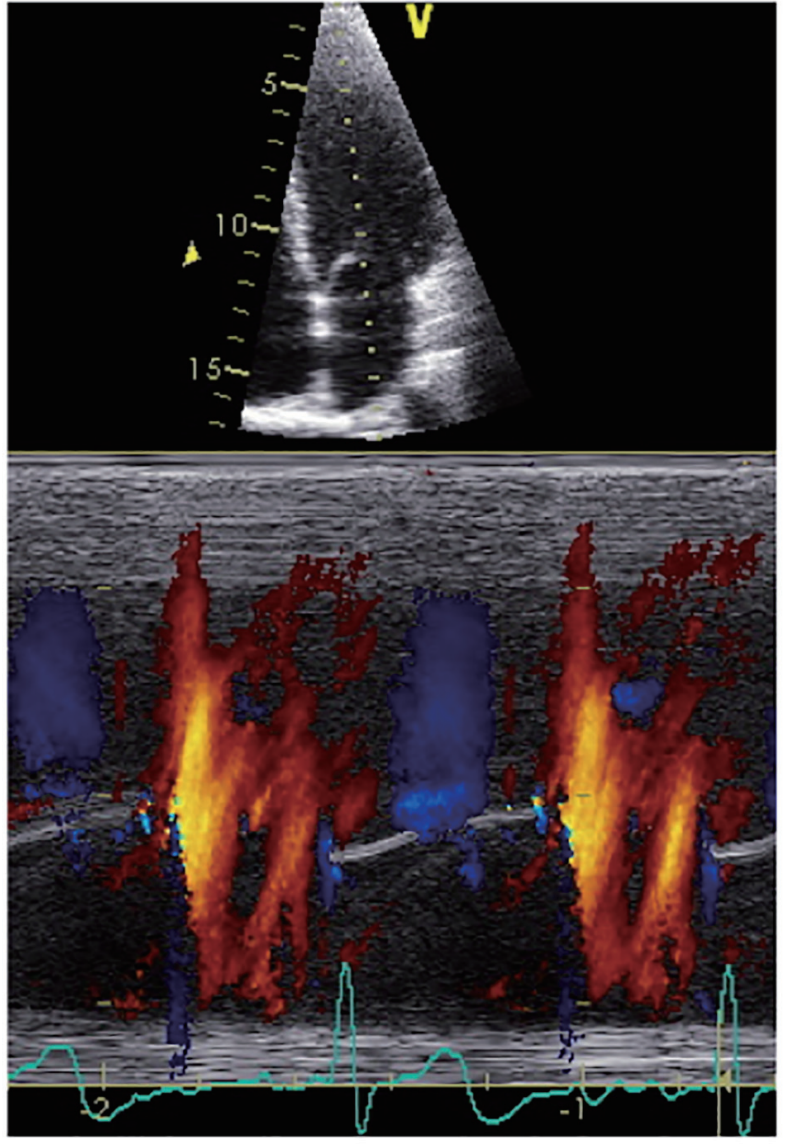

Figure 1. A representative normal color $\mathrm{M}$-mode image is shown with a temporal resolution of approximately $5 \mathrm{~ms}$, a spatial resolution (depth) of approximately $1 \mathrm{~mm}$ and a velocity resolution of approximately 4 $\mathrm{cm} / \mathrm{s}$, according to the Nyquist limit. The resultant spatio-temporal velocity information is expressed as a distance to time ratio more commonly expressed as $\mathrm{cm} / \mathrm{s}$.

the apical four-chamber view. LV diastolic pressures were estimated using the mitral valve inflow E/mitral annular TDI $\mathrm{E}^{\prime}$ ratio [16]. Finally, LV diastolic function was classified as normal, impaired relaxation, or pseudonormal pattern following published recommendations [16]. 4) Color M-mode acquisitions were made from the four-chamber view during transthoracic apical imaging. Direction of the inflowing blood is identified by placing a large color box from the mitral valve to the apex and care is taken to assure that the scan line goes through the center of the mitral valve and along the central part of the blood column (staying within the $60 \%$ central part of the mitral orifice and limiting misalignment to $<20^{\circ}$ is acceptable) $[8,9]$. Gain is set at sub-saturation levels and the Nyquist range limit is adapted to $\pm 75 \%$ of the spectral E velocity to obtain overflow ("aliasing"). Switching to M-mode and with the chart recorder set at a sweep rate of $100 \mathrm{~mm} / \mathrm{s}$, an M-mode spatio-temporal velocity map with the shape of a "flame" is displayed, as shown in Figure 1.

From the $\mathrm{Vp}$ data then the following quantitative information was obtained. First, a Vp value between 55 and $45 \mathrm{~cm} / \mathrm{s}$ is used to define impaired relaxation in the young, and mid-aged 
Table 1. Relevant Echocardiographic Data of the Studied Population

\begin{tabular}{llllll}
\hline Variables & Group I $(\mathbf{n}=\mathbf{1 5})$ & Group II $(\mathbf{n}=\mathbf{2 7})$ & Group III $(\mathbf{n}=\mathbf{2 7})$ & Group IV $(\mathbf{n}=\mathbf{6 6})$ & P-value \\
\hline LVESV $\left(\mathrm{cm}^{3}\right)$ & $40 \pm 15$ & $40 \pm 27$ & $73 \pm 54^{\S}$ & $34 \pm 17$ & $<0.01$ \\
LVEDV $\left(\mathrm{cm}^{3}\right)$ & $119 \pm 30$ & $112 \pm 30$ & $145 \pm 66^{\boldsymbol{⿰}}$ & $81 \pm 35^{\beta}$ & $<0.01$ \\
LVEF $(\%)$ & $67 \pm 7$ & $67 \pm 13$ & $57 \pm 13^{\S}$ & $59 \pm 9^{\beta}$ & $<0.01$ \\
MA E/A ratio & $1.3 \pm 0.3$ & $0.8 \pm 0.1^{\dagger}$ & $1.7 \pm 0.7^{\S}$ & $1.2 \pm 0.6$ & $<0.01$ \\
MV E/MA E' ratio & $7 \pm 3$ & $9 \pm 3$ & $17 \pm 6^{\S}$ & $10 \pm 12$ & $<0.01$ \\
\hline
\end{tabular}

LV: left ventricle; LVESV: LV end-systolic volume; LVEDV: LV end-diastolic volume; LVEF: LV ejection fraction; MA: mitral annulus; MV: mitral valve. ${ }^{\dagger}$ Bonferroni test indicates significant differences $(\mathrm{P}<0.05)$ between group II and groups I, III, IV. TGroup III and group II, §Group III and groups I, II, IV and ${ }^{\beta}$ Group IV and groups I, II, II.

adults, respectively $[10,14,19,24-27]$. Second, Vp has also been found useful to estimate LV filling pressures using the following formula that has been used to calculate pulmonary capillary wedge pressure $(\mathrm{PCWP})$ : $\mathrm{PCWP}=(5.27 \times \mathrm{E} / \mathrm{Vp})+4.6$.

With this formula, a value for $\mathrm{E} / \mathrm{Vp}>1.5$ has been shown to predict a PCWP $>12 \mathrm{~mm} \mathrm{Hg}$ in a heterogeneous group that comprises normal, ischemic, hypertrophic and dilated hearts with positive and negative predictive values of $93 \%$ and $70 \%$, respectively $[28,29]$.

\section{Statistical analysis}

All echocardiographic parameters were calculated using the commercially available software Merge Cardio Workstation (Merge Healthcare). Continuous variables were compared between all groups using one-way analysis of variance (ANOVA) with Bonferroni correction, assuming equal variances. In addition, simple linear regression analysis was the preferred method to determine the association between continuous variables. A P-value of less than 0.05 was considered statistically significant. All statistics were calculated in MedCalc Software bvba Version 14.12.0 (Belgium).

\section{Results}

The study population included 135 patients that were divided into four groups. Group I included 15 patients with a normal LV diastolic function (mean age $41 \pm 11$ years, $60 \%$ males). Group II consisted of 27 patients classified as LVDD stage 1 (mean age $59 \pm 12$ years, 67\% males). Group III included 27 patients classified as LVDD stage 2 (mean age $57 \pm 13$ years, $54 \%$ females). Group IV was composed of 66 patients with known chronic PAH by prior heart catheterization (mean age $58 \pm 12$ years, $83 \%$ females). None of the individuals of groups I, II and III had any grade of PAH. While patients in group I were younger any other three groups, as expected, there was no statistical difference in terms of age between the two LVDD and PAH groups $(\mathrm{P}>0.05)$. Furthermore, most of the patients in both groups I and II were males while females predominated in groups II and IV. Finally, there was no statistical difference in terms of body surface area (BSA) among the studied groups (group I: $1.96 \pm 0.33$; group II: $1.98 \pm 0.24$; group III: $2.11 \pm$ 0.38 ; group IV: $2.01 \pm 0.31 ; \mathrm{P}=0.067$ ).
With regard to the relevant echocardiographic data collected for this study as seen in Table 1, the largest end-systolic as well as end-diastolic LV volumes were found in group III patients. Similarly, the smaller end-diastolic LV volumes were noted in PAH (group IV) patients. Also, LV ejection fraction (EF) was lowest in group III and IV when compared to the other two groups. Finally, both MA E'/A' and MV E/MA E' ratios were used to confirm the LVDD classification.

When we first examined Vp values (Fig. 2a), as expected, the lowest $\mathrm{Vp}$ values were noted in both LVDD patient groups (II and III) when compared to patients with normal LV diastolic function $(\mathrm{P}<0.05)$. In contrast, the highest $\mathrm{Vp}$ values were recorded in PAH (group IV) patients when compared to the other three groups $(\mathrm{P}<0.05)$. Additionally, in terms of PCWP, the highest values were found on group II: $15 \pm 4 \mathrm{~mm} \mathrm{Hg}$ and group III: $21 \pm 5 \mathrm{~mm} \mathrm{Hg}(\mathrm{P}<0.05)$ when compared to patients with normal LV diastolic function (group I: $12 \pm 3 \mathrm{~mm} \mathrm{Hg}$ ) as well as PAH patients (group IV: $11 \pm 3 \mathrm{~mm} \mathrm{Hg}$ ). As expected, the highest PCWP values were found in group III patients $(\mathrm{P}<$ 0.05) (Fig. 2b). Representative color M-mode Vp images for all four studied groups are shown on Figure 3.

To better explore the association between Vp and PCWP, MA TDI E', MV E/MA E' ratio as well as MV E/Vp and MV E/MA E' ratios, a simple linear regression was performed. Interestingly, we found that LVVp was inversely associated with PCWP (Fig. 4). However, this association was just significant in patients with LV dysfunction or pulmonary hypertension (PH) (Table 2). Additionally, the relationship between MV E/ Vp and MV E/MA E' was positive and significant for all study groups $(\mathrm{P}<0.01)$.

\section{Discussion}

Our study is firstly, to our knowledge, aimed directly at comparing $\mathrm{Vp}$ values obtained from a heterogeneous group of PAH patients to $\mathrm{Vp}$ values acquired from patients with both normal and abnormal LV diastolic function (stages 1 and 2). From our analysis, we found that the highest $\mathrm{Vp}$ values are recorded from PAH patients while the lowest Vp values were seen in LVDD patients.

On a typical color M-mode tracing, Vp data are displayed along a scan line with a temporal resolution of $5 \mathrm{~ms}$, a spatial resolution of about $1 \mathrm{~mm}$, and a velocity resolution equal to the forward plus the reversed Nyquist limit divided by 32 [12]. In 

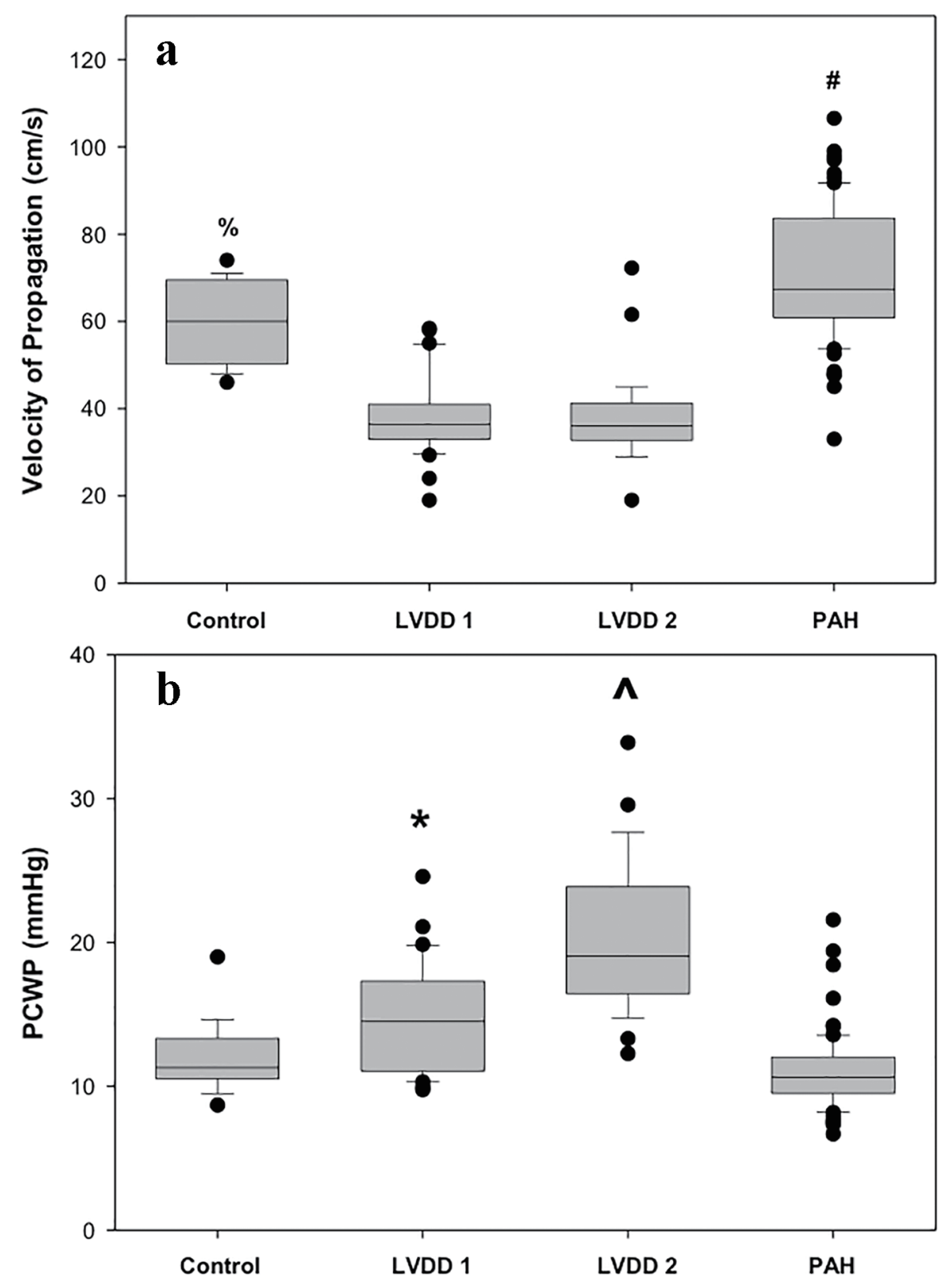

Figure 2. (a) Box plot representation of $V p$ values from all four groups. Bonferroni test indicates significant differences $(P<0.05)$ between: (\#) PAH and control, LVDD 1, LVDD 2 and (\%) control and LVDD 1, LVDD 2 groups. (b) Box plot representation of PCWP values from all four groups. Bonferroni test indicates significant differences $(P<0.05)$ between: $\left({ }^{\wedge}\right)$ LVDD 2 and control, LVDD 1, PAH and $\left(^{*}\right)$ LVDD 1 and control, PAH groups.

a typical color M-mode tracing while the patient is in normal sinus rhythm, the first wave propagates from the atria to the ventricular apex corresponding to early filling and a second wave follows atrial contraction. The magnitude of these velocities is highest above the valve leaflet tips and decreases as flow approaches the apex, as shown by the change in encoding color. Additionally, in normal ventricles, the spatial position of the maximal velocity is closer to the ventricular apex for the early filling wave than it is for the atrial contraction wave, suggesting that intraventricular pressure gradients during early filling produce a suction force that accelerates flow beyond the valve orifice $[12,14]$.

The utility of color M-mode interrogation across the mitral valve depicting $\mathrm{Vp}$ data has been useful in terms of assessing LV diastolic function. Initial validation data, published by Garcia et al, demonstrated that color M-mode Doppler Vp data not only were unaffected by preload alterations, but also mainly dependent on LV relaxation [13]. Furthermore, Stewart and associates analyzing color M-mode propagation velocities in patients with varying degrees of LVDD found that earlydiastolic flow propagation occurs with an initial rapid velocity that abruptly decelerates to a terminal velocity [15]. Most im- 

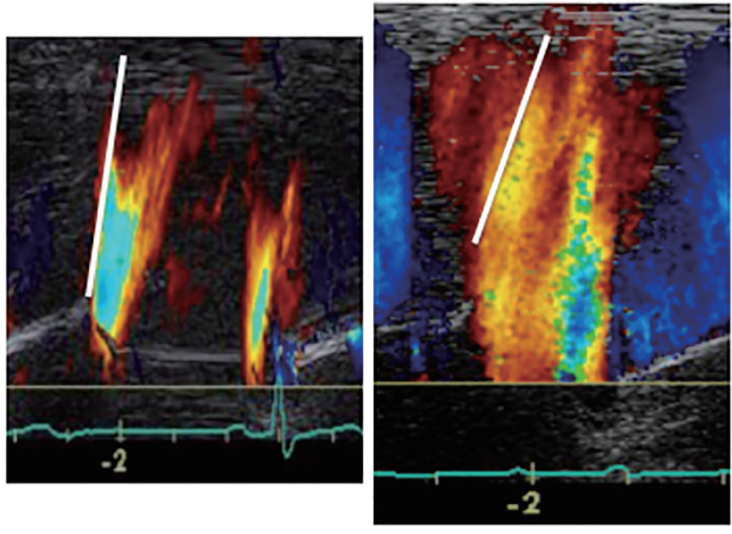

Group I

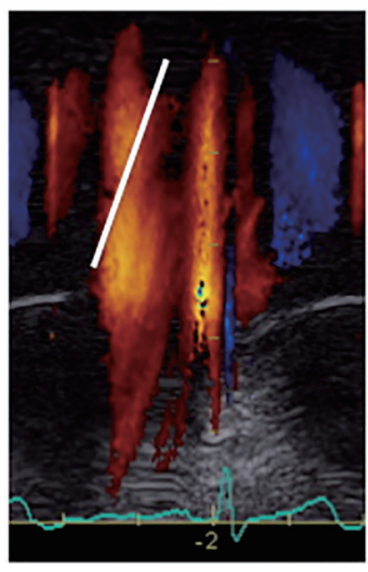

Group III

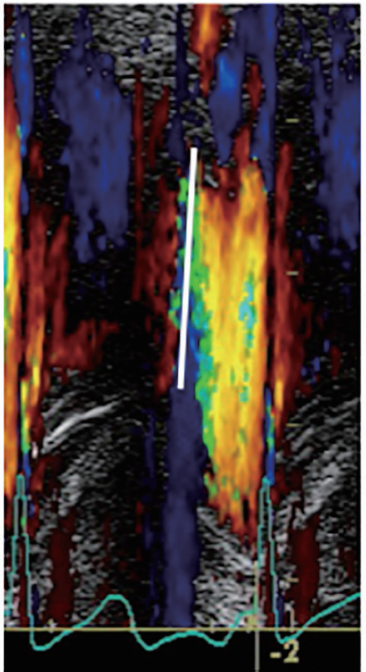

Group IV

Figure 3. Representative color M-mode Vp images for the four studied groups.

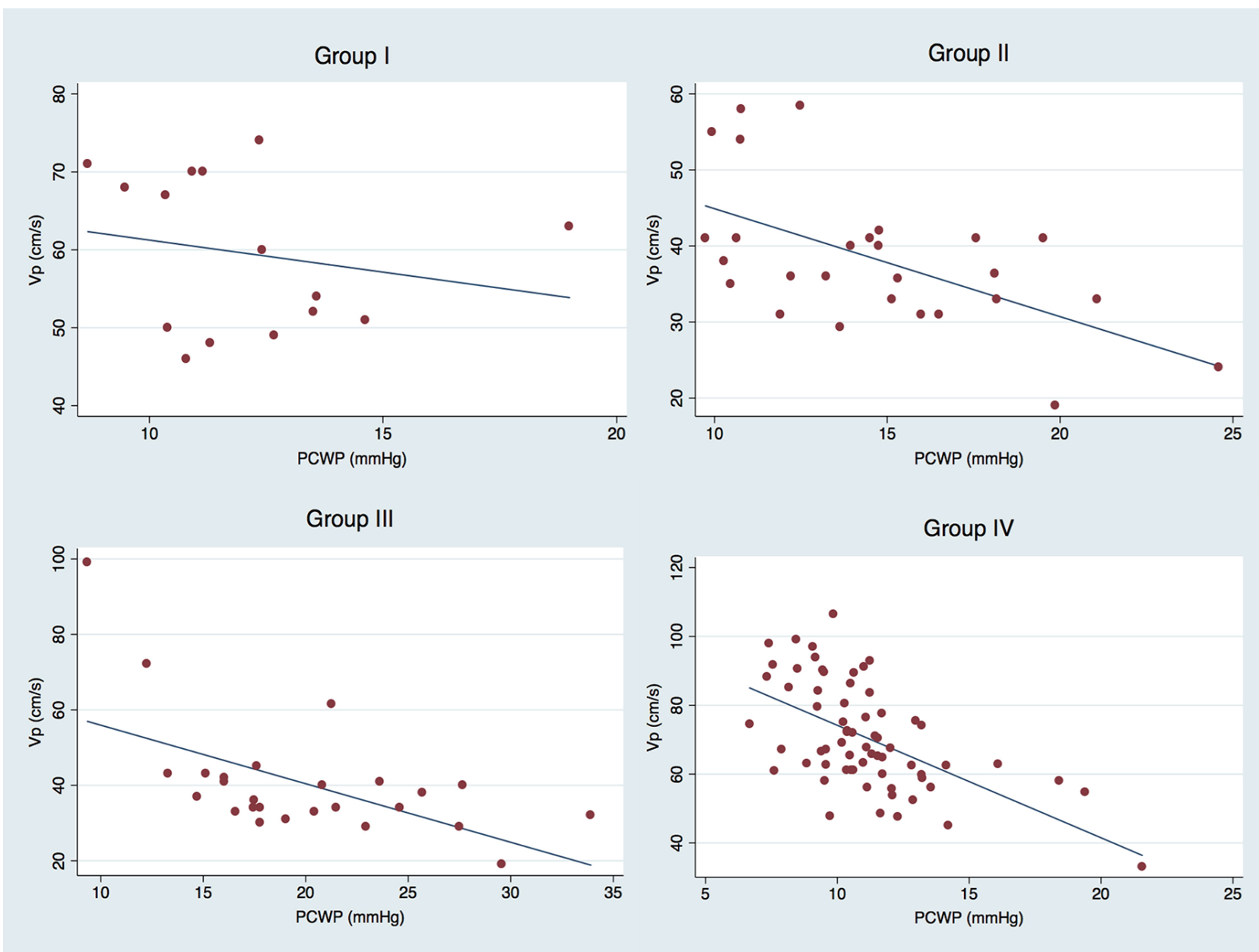

Figure 4. Scatter plot graphs showing the relationship between Vp and PCWP for all study groups. 
Table 2. Simple Linear Regression Analysis to Determine the Association Between Important Echocardiographic Variables

\begin{tabular}{lllll}
\hline \multirow{2}{*}{ Variables } & \multicolumn{3}{c}{ Coefficient (P-value) } \\
\cline { 2 - 5 } & Group I & Group II & Group III & Group IV \\
\hline Vp vs. PCWP & $-0.8213(0.46)$ & $-1.4200(<0.01)$ & $-1.5520(<0.01)$ & $-3.2673(<0.01)$ \\
Vp vs. MV E/MA E' ratio & $1.1685(0.22)$ & $-0.8431(0.12)$ & $-0.2637(0.64)$ & $-0.2192(0.18)$ \\
Vp vs. MA E' & $-0.5117(0.56)$ & $1.9742(0.02)$ & $2.2038(0.29)$ & $-0.1049(0.41)$ \\
MV E/Vp vs. MV E/MA E' ratio & $0.1188(<0.01)$ & $0.1416(<0.01)$ & $0.1257(<0.01)$ & $0.0146(<0.01)$ \\
\hline
\end{tabular}

Vp: velocity of propagation; PCWP: pulmonary capillary wedge pressure; MA: mitral annulus; MV: mitral valve.

portantly, they also noted that with LVDD the initial velocity is slower and the deceleration point occurs closer to the MA than with normal filling.

Even though the American Society of Echocardiography identified Vp not only as a semiquantitative marker of LVDD, but also its use in conjunction with mitral $\mathrm{E}$ as useful in predicting LV filling pressures in a previous publication [5], the new guidelines point out that $\mathrm{Vp}$ though reliable as an index of LV relaxation in patients with depressed LV systolic function and dilated LV was not so useful in patients with normal LV systolic function [16]. Furthermore, the American Society of Echocardiography has now issued some comments regarding $\mathrm{Vp}$ due to lower feasibility and reproducibility as well as problems with angulation between M-mode cursor and flow that might lead to erroneous measurements.

Notwithstanding $\mathrm{Vp}$ values have not been previously compared between PAH and patients with LVDD, our laboratory has used color M-mode to study if there were any differences between the temporal onset of the Vp signal between individuals with normal pulmonary artery systolic pressures and patients with chronic PAH [30]. In this study, we found that chronic PAH not only disrupted RV and LV diastolic Vp signal distribution, but also their temporal occurrence. These findings implied the presence of significant interventricular diastolic dyssynchrony as a result of PAH.

From a mechanistic point of view RV remodeling is known to occur as a result of an increased afterload in PAH. During this adaptive process RV hypertrophy initially occurs, followed by RV dilation [31]. Once the RV dilates not only RV contractility is compromised, but also this causes further RV dilatation due to tricuspid regurgitation. Since the RV and LV do not function in isolation and share a common pericardial sac and interventricular septum, the leftward ventricular septal bowing that occurs with PAH not only leads to LV underfilling $[32,33]$, but also impaired LV contractility occurs through ventricular interdependence [32]. Reduction in early LV diastolic filling velocity documented by cardiac magnetic resonance imaging has been clearly shown as a result of an abnormal intraventricular septal deformation toward the LV cavity [34, 35]. Our group has previously shown that this abnormal systolic eccentricity motion is the best echocardiographic predictor of $\mathrm{PH}$ [36]. Therefore, the results of the present study seem to align with the known functional and anatomical changes occurring as a result of PAH resulting in significantly higher Vp values. In fact, we have previously described a case report of a patient with severe PAH whose propagation velocity was almost perpendicular after Valsalva, resembling the propagation velocity profile of patients with constrictive pericarditis [37].

The presence of both RV systolic and diastolic dyssynchrony has been shown to occur in PAH patients [30, 38, 39]; however, LV diastolic flows have not been either well studied or characterized. Therefore, these results underscore the need to broaden our attention when studying the effects of PAH, so that we can better understand our findings in lieu of clinical symptoms and overall prognosis.

The following limitations need to be acknowledged. First, this was a retrospective study; however, the main goal was attained. Second, a small number of patients were included for analysis. However, the main purpose of this study was to determine if there were any difference in $\mathrm{Vp}$ values from $\mathrm{PAH}$ patients when compared to patients with normal LV diastolic function as well as with patients with stage 1 and 2 LVDD and this was demonstrated. Third, based on the pre-specified exclusion criteria no assumptions can be made on how atrial fibrillation or frequent ectopy could affect the reliability of our study findings. Fourth, RV dimensions were not available from the patients with LVDD to make any potential correlations between right-sided chamber size and Vp values. Even though color Mmode is only known to assess parameters in two dimensions and at first glance might be considered inadequate to describe diastolic flows, as these flows occur as vortices and consequently would be better assessed using a three-dimensional mode; the intent of this work was to evaluate the influence of PAH on LV diastolic Vp. Future studies should now determine the relationship that might exist between $\mathrm{Vp}$ and right-sided cavity sizes as a result of PAH. Fifth, lack of invasive hemodynamic data might be considered as a limitation. However, the main study goal was met. With these results we can now propose future studies that should address if potential correlations can be drawn between invasive hemodynamic data and echocardiographic color M-mode Vp values. It can also be argued that we lack sequential echocardiographic studies on $\mathrm{PAH}$ patients to validate the utility of $\mathrm{Vp}$ findings over time. Finally, some might argue that intraobserver as well as interobserver variability results were not obtained. Even though we have previously published these results for color M-mode $\mathrm{Vp}$ measurements for both RV and LV [30], we do acknowledge the potential limitation of using color M-mode to study diastolic flows.

\section{Conclusion}

In summary, this study suggests that PAH patients show significantly higher $\mathrm{Vp}$ values when compared to patients with 
both, normal and abnormal LV diastolic function (stages 1 and 2). These results not only might reflect the compressive forces being exerted on the LV by the dilated and hypertrophied RV, known to increase $\mathrm{Vp}$ but also might be useful for non-invasive assessment of PCWP in PAH patients. Additional prospective studies are now warranted to assess its clinical utility.

\section{Acknowledgments}

This publication was partially supported by the National Institute on Minority Health and Health Disparities of the National Institutes of Health Award Numbers CCTRECDR25MD007607 and HiREC-S21MD001830. Its contents are solely the responsibility of the authors and do not necessarily represent the official views of the National Institutes of Health.

\section{Conflicts of Interest}

The authors report no conflicts of interest.

\section{References}

1. Vasan RS, Levy D. Defining diastolic heart failure: a call for standardized diagnostic criteria. Circulation. 2000;101(17):2118-2121.

2. Vasan RS, Benjamin EJ, Levy D. Prevalence, clinical features and prognosis of diastolic heart failure: an epidemiologic perspective. J Am Coll Cardiol. 1995;26(7):15651574.

3. Zile MR, Brutsaert DL. New concepts in diastolic dysfunction and diastolic heart failure: Part I: diagnosis, prognosis, and measurements of diastolic function. Circulation. 2002;105(11):1387-1393.

4. De Boeck BW, Oh JK, Vandervoort PM, Vierendeels JA, van der Aa RP, Cramer MJ. Colour M-mode velocity propagation: a glance at intra-ventricular pressure gradients and early diastolic ventricular performance. Eur J Heart Fail. 2005;7(1):19-28.

5. Nagueh SF, Appleton CP, Gillebert TC, Marino PN, Oh JK, Smiseth OA, Waggoner AD, et al. Recommendations for the evaluation of left ventricular diastolic function by echocardiography. Eur J Echocardiogr. 2009;10(2):165193.

6. Katz LN. The role played by the ventricular relaxation process in filling the ventricle. Am J Physiol. 1930;95:542553.

7. Djaiani GN, McCreath BJ, Ti LK, Mackensen BG, Podgoreanu M, Phillips-Bute B, Mathew JP. Mitral flow propagation velocity identifies patients with abnormal diastolic function during coronary artery bypass graft surgery. Anesth Analg. 2002;95(3):524-530, table of contents.

8. Greenberg NL, Castro PL, Drinko J, Garcia MJ, Thomas JD. Effect of scanline orientation on ventricular flow propagation: assessment using high frame-rate color Doppler echocardiography. Biomed Sci Instrum. 2000;36:203208.

9. Greenberg NL, Vandervoort PM, Firstenberg MS, Garcia MJ, Thomas JD. Estimation of diastolic intraventricular pressure gradients by Doppler M-mode echocardiography. Am J Physiol Heart Circ Physiol. 2001;280(6):H25072515.

10. Stugaard M, Smiseth OA, Risoe C, Ihlen H. Intraventricular early diastolic filling during acute myocardial ischemia, assessment by multigated color m-mode Doppler echocardiography. Circulation. 1993;88(6):2705-2713.

11. Sessoms MW, Lisauskas J, Kovacs SJ. The left ventricular color M-mode Doppler flow propagation velocity $\mathrm{V}(\mathrm{p})$ : in vivo comparison of alternative methods including physiologic implications. J Am Soc Echocardiogr. 2002;15(4):339-348.

12. Thomas JD, Garcia MJ, Greenberg NL. Application of color Doppler M-mode echocardiography in the assessment of ventricular diastolic function: potential for quantitative analysis. Heart Vessels. 1997;(Suppl 12):135-137.

13. Garcia MJ, Smedira NG, Greenberg NL, Main M, Firstenberg MS, Odabashian J, Thomas JD. Color M-mode Doppler flow propagation velocity is a preload insensitive index of left ventricular relaxation: animal and human validation. J Am Coll Cardiol. 2000;35(1):201-208.

14. Garcia MJ, Thomas JD, Klein AL. New Doppler echocardiographic applications for the study of diastolic function. J Am Coll Cardiol. 1998;32(4):865-875.

15. Stewart KC, Kumar R, Charonko JJ, Ohara T, Vlachos PP, Little WC. Evaluation of LV diastolic function from color M-mode echocardiography. JACC Cardiovasc Imaging. 2011;4(1):37-46.

16. Nagueh SF, Smiseth OA, Appleton CP, Byrd BF, 3rd, Dokainish H, Edvardsen T, Flachskampf FA, et al. Recommendations for the Evaluation of Left Ventricular Diastolic Function by Echocardiography: An Update from the American Society of Echocardiography and the European Association of Cardiovascular Imaging. J Am Soc Echocardiogr. 2016;29(4):277-314.

17. Nikolic SD, Feneley MP, Pajaro OE, Rankin JS, Yellin EL. Origin of regional pressure gradients in the left ventricle during early diastole. Am J Physiol. 1995;268(2 Pt 2):H550-557.

18. Steine K, Stugaard M, Smiseth OA. Mechanisms of retarded apical filling in acute ischemic left ventricular failure. Circulation. 1999;99(15):2048-2054.

19. Firstenberg MS, Smedira NG, Greenberg NL, Prior DL, McCarthy PM, Garcia MJ, Thomas JD. Relationship between early diastolic intraventricular pressure gradients, an index of elastic recoil, and improvements in systolic and diastolic function. Circulation. 2001;104(12 Suppl 1):I330-335.

20. Shortland AP, Black RA, Jarvis JC, Henry FS, Iudicello F, Collins MW, Salmons S. Formation and travel of vortices in model ventricles: application to the design of skeletal muscle ventricles. J Biomech. 1996;29(4):503-511.

21. Bristow MR, Zisman LS, Lowes BD, Abraham WT, Badesch DB, Groves BM, Voelkel NF, et al. The pressureoverloaded right ventricle in pulmonary hypertension. 
Chest. 1998;114(1 Suppl):101S-106S.

22. Marcus JT, Vonk Noordegraaf A, Roeleveld RJ, Postmus PE, Heethaar RM, Van Rossum AC, Boonstra A. Impaired left ventricular filling due to right ventricular pressure overload in primary pulmonary hypertension: noninvasive monitoring using MRI. Chest. 2001;119(6):17611765.

23. Lang RM, Badano LP, Mor-Avi V, Afilalo J, Armstrong A, Ernande L, Flachskampf FA, et al. Recommendations for cardiac chamber quantification by echocardiography in adults: an update from the American Society of Echocardiography and the European Association of Cardiovascular Imaging. J Am Soc Echocardiogr. 2015;28(1):139 e14.

24. Takatsuji H, Mikami T, Urasawa K, Teranishi J, Onozuka $\mathrm{H}$, Takagi C, Makita Y, et al. A new approach for evaluation of left ventricular diastolic function: spatial and temporal analysis of left ventricular filling flow propagation by color M-mode Doppler echocardiography. J Am Coll Cardiol. 1996;27(2):365-371.

25. Stugaard M, Risoe C, Ihlen H, Smiseth OA. Intracavitary filling pattern in the failing left ventricle assessed by color M-mode Doppler echocardiography. J Am Coll Cardiol. 1994;24(3):663-670.

26. Steine K, Flogstad T, Stugaard M, Smiseth OA. Early diastolic intraventricular filling pattern in acute myocardial infarction by color M-mode Doppler echocardiography. J Am Soc Echocardiogr. 1998;11(2):119-125.

27. Nishihara K, Mikami T, Takatsuji H, Onozuka H, Saito N, Yamada S, Urasawa K, et al. Usefulness of early diastolic flow propagation velocity measured by color M-mode Doppler technique for the assessment of left ventricular diastolic function in patients with hypertrophic cardiomyopathy. J Am Soc Echocardiogr. 2000;13(9):801-808.

28. Garcia MJ, Ares MA, Asher C, Rodriguez L, Vandervoort $\mathrm{P}$, Thomas JD. An index of early left ventricular filling that combined with pulsed Doppler peak E velocity may estimate capillary wedge pressure. J Am Coll Cardiol. 1997;29(2):448-454.

29. Firstenberg MS, Levine BD, Garcia MJ, Greenberg NL, Cardon L, Morehead AJ, Zuckerman J, et al. Relationship of echocardiographic indices to pulmonary capillary wedge pressures in healthy volunteers. J Am Coll Cardiol. 2000;36(5):1664-1669.

30. Lopez-Candales A, Edelman K. Chronic pulmonary hy- pertension causes significant interventricular spatiotemporal dyssynchrony when onset of diastolic flow signals are assessed by color m-mode. Echocardiography. 2012;29(6):653-660.

31. Vonk Noordegraaf A, Galie N. The role of the right ventricle in pulmonary arterial hypertension. Eur Respir Rev. 2011;20(122):243-253.

32. Hardegree EL, Sachdev A, Fenstad ER, Villarraga HR, Frantz RP, McGoon MD, Oh JK, et al. Impaired left ventricular mechanics in pulmonary arterial hypertension: identification of a cohort at high risk. Circ Heart Fail. 2013;6(4):748-755.

33. Motoji Y, Tanaka H, Fukuda Y, Sano H, Ryo K, Imanishi $\mathrm{J}$, Miyoshi T, et al. Interdependence of right ventricular systolic function and left ventricular filling and its association with outcome for patients with pulmonary hypertension. Int J Cardiovase Imaging. 2015;31(4):691-698.

34. Roeleveld RJ, Marcus JT, Faes TJ, Gan TJ, Boonstra A, Postmus PE, Vonk-Noordegraaf A. Interventricular septal configuration at mr imaging and pulmonary arterial pressure in pulmonary hypertension. Radiology. 2005;234(3):710-717.

35. Marcus JT, Gan CT, Zwanenburg JJ, Boonstra A, Allaart $\mathrm{CP}$, Gotte MJ, Vonk-Noordegraaf A. Interventricular mechanical asynchrony in pulmonary arterial hypertension: left-to-right delay in peak shortening is related to right ventricular overload and left ventricular underfilling. J Am Coll Cardiol. 2008;51(7):750-757.

36. Lopez-Candales A, Rajagopalan N, Kochar M, Gulyasy B, Edelman K. Systolic eccentricity index identifies right ventricular dysfunction in pulmonary hypertension. Int $\mathbf{J}$ Cardiol. 2008;129(3):424-426.

37. Lopez-Candales A, Cleary H, Edelman K. Constrictive-like physiology in severe pulmonary hypertension: the effect of the Valsalva maneuver. South Med J. 2011;104(4):282-284.

38. Lopez-Candales A, Dohi K, Bazaz R, Edelman K. Relation of right ventricular free wall mechanical delay to right ventricular dysfunction as determined by tissue Doppler imaging. Am J Cardiol. 2005;96(4):602-606.

39. Lopez-Candales A, Dohi K, Rajagopalan N, Suffoletto M, Murali S, Gorcsan J, Edelman K. Right ventricular dyssynchrony in patients with pulmonary hypertension is associated with disease severity and functional class. Cardiovasc Ultrasound. 2005;3:23. 